\title{
Planning Hand-Arm Grasping Motions with Human-Like Appearance
}

\author{
Néstor García, Raúl Suárez and Jan Rosell
}

\begin{abstract}
This paper addresses the problem of obtaining human-like motions on hand-arm robotic systems performing pick-and-place actions. The focus is set on the coordinated movements of the robotic arm and the anthropomorphic mechanical hand, with which the arm is equipped. For this, human movements performing different grasps are captured and mapped to the robot in order to compute the human hand synergies. These synergies are used to reduce the complexity of the planning phase by reducing the dimension of the search space. In addition, the paper proposes a sampling-based planner, which guides the motion planning following the synergies. The introduced approach is tested in an application example and thoroughly compared with other state-of-the-art planning algorithms, obtaining better results.
\end{abstract}

\section{INTRODUCTION}

Nowadays, robots are turning essential in more fields and applications, thanks to becoming adapted to different tasks and environments but also getting more sophisticated and complex. The humanoid robots equipped with anthropomorphic dexterous hands are one of the most representative examples. In fact, these mechanical hands are devices that concentrate in a compact volume a high number of sensors and degrees of freedom (DOFs), ranging usually from 12 to 25 DOFs. In addition, despite all these advanced features, the automatic planning of their movements must be solved to obtain a satisfactory performance, which is still an arduous and non-evident task since the complexity of the problem increases exponentially with the number of DOFs. Furthermore, sometimes not only a feasible path is required but also the one that optimizes some path quality metric (e.g. minimizing the path length, the execution time or the energy consumption). Regarding the humanoid robotics, for instance, the motion planning must not only focus on the efficient search of a valid solution, but also on the search of robot movements that mimic the motions of the human beings. Pursuing this goal, the human-robot collaboration is facilitated because, thereby, the humans can adjust their motions to avoid possible injuries or enhance the collaboration since they are familiar with the robot motions [1].

On the one hand, the motion planning of complex systems has been typically addressed with different planning algorithms, being the sampling-based planners [2] and, especially among them, the Probabilistic Roadmap planners, PRM [3], and the Rapidly-exploring Random Trees, RRT [4], the most

The authors are with the Institute of Industrial and Control Engineering (IOC), Universitat Politècnica de Catalunya (UPC), Barcelona, Spain (\{nestor.garcia.hidalgo, jan.rosell, raul.suarez\}@upc.edu). This work was partially supported by the Spanish Government through the project DPI2016-80077-R. Besides, N. García is supported by the Generalitat de Catalunya through the grant FI-DGR 2018. commonly used. These algorithms have been researched extensively and, hence, several variants exist, for instance to deal with constraints [5], or to bias the sampling towards better regions of the configuration space by using, potential fields [6] or retraction-based methods [7].

On the other hand, the robot joints must be properly coordinated in order to obtain human-like motions. Thereby, real human movements are commonly used as a reference [8], either pursuing a direct on-line teleoperation of the robot [9], or with the aim of analyzing these movements and getting some valuable information to be applied later in a planning phase [10]. Some relevant pioneering works dealt with the grasping problem analyzing the correlations of the finger joints when the human hand was grasping objects [11]. These correlations were called hand postural synergies and mapped into a mechanical hand [12]. The synergies existing in the human hand were also used for other objectives such as the analysis and design of robotic hands in order to mimic human grasps [13], the design of specific hand control systems [14], or the identification of the hand pose using low-cost gloves [15]. Nevertheless, there exist other approaches that, instead of studying the hand synergies while grasping an object, compute the synergies from hand movements when the human tries to cover the whole hand configuration space in an unconstrained way [16]. More recently, a compliant model, called soft synergies, was also introduced and used in the selection of grasping forces, in their control, and in the control of the motion of the grasped object [17], [18]. In addition, the synergies were used in a dual-arm anthropomorphic system while performing manipulation tasks [10], [19]. The works mentioned above dealt with synergies involving correlations between joint positions. Nevertheless, a recent work extended the concept of synergies to the velocity space (i.e. the space of the first derivative of the configuration trajectories) calling them first-order synergies [20], [21] (in contrast with the synergies in the configuration space, that were called zero-order synergies).

This work proposes to characterize the synergies existing in the human grasping motion, considering the different grasp types and grasp phases, and then use a sampling-based motion planner, especially designed to use these synergies, in order reduce the planning complexity and at the same time look for hand-arm movements with human-like appearance.

After this introduction, Section II presents the problem statement and gives an overview of the proposed approach, Section III details the proposal, the approach is validated in Section IV and finally Section V presents the conclusions and future work. 

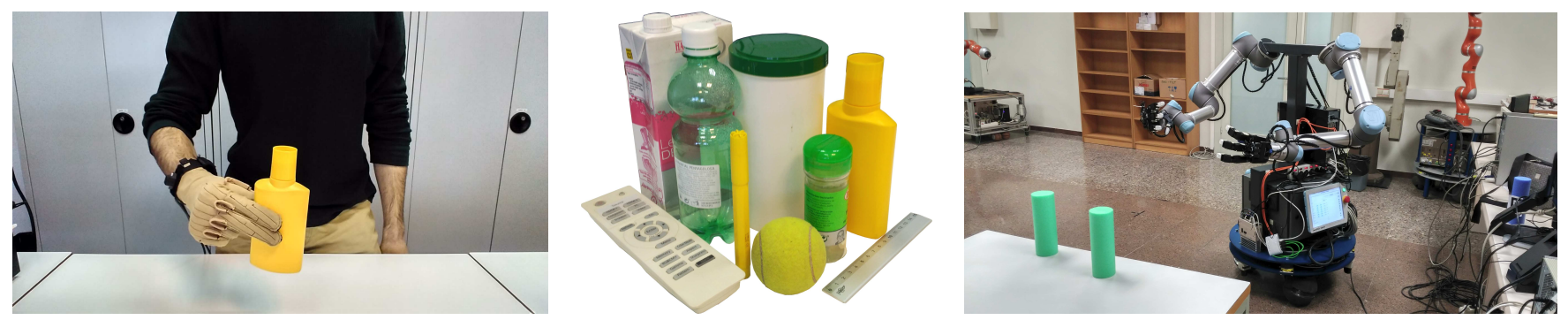

Fig. 1. Human operator wearing the measurement equipment (left), set of grasped objects in the experiments (middle), and dual-arm robot (right).

\section{PRoblem STATEMENT AND APPROACH OVERVIEW}

The goal of this work is to plan the motions of a hand-arm robotic system robot trying to mimic the hand-arm movements that a human does to pick an object performing different grasps. To this end, a sampling-based planning algorithm is designed and the movements of a human operator are used to guide the motion planning. The main features of the proposed approach are the following:

1) The motions of a human operator performing different grasps on several objects are captured, and then mapped to the robot whose motions are aimed to be planned (see Fig. 1). Thereby, the synergies existing in the human motions when a given grasp is done are computed.

2) The computed synergies are used to guide the motion planning and to reduce the complexity of the planning phase through a reduction of the dimension of the search space, being this dimension-reduction process dependent on the grasp type to perform.

3) A bidirectional sampling-based planner is designed to use the proposed dimension-reduction method and to bias the tree growth towards the directions of the computed synergies. Hence, human-like movements are obtained with a low computational load.

\section{PlanNing PROCEDURE}

\section{A. Motion capture and mapping}

In this work, human motions are used as a reference to obtain human-like movements of a hand-arm robotic system picking a given object. Many types of common human grasps are gathered in the grasp taxonomy of M.R. Cutkosky [22], which classifies the grasps depending on the object size and on the dexterity of the task to perform with the grasped object. Although this classification is not complete, and there exists more extensive grasp classifications (e.g. [23]), it is detailed enough for the considered purposes. Besides, W. Dai, Y. Sun and X. Qian [24] updated the taxonomy of Cutkosky and analyzed, from a different perspective, the entire grasping trajectory and not only the grasping configuration (i.e. the final snapshot), proving that the grasp types can be grouped naturally into consistent grasp families (see Fig. 2). This family-grouping is used here to adapt the planning process according to the grasp being performed (even though several potential grasp types are considered simultaneously).
Thereby, using a Cyberglove sensorized glove with a $50 \mathrm{~Hz}$ sampling frequency, the motions of a human operator are recorded performing 15 different grasp types on 9 objects, with 12 repetitions per grasp type and starting off from a comfortable stand position in front of the object (see Fig. 1 and 2). This implies 180 demonstrations and more than 15000 configuration samples (where each sample contains 22 measurements describing the positions of the finger joints read from the glove). Once the samples have been captured, they are mapped to the robotic hand. This mapping depends on the kinematic structure and particularities of the used robotic system. In this work, a robotic hand-arm system composed of a 6-DOF UR5 robotic arm equipped with a 16-DOF Allegro Hand is used (see Fig. 1). On the one hand, the information regarding the little finger is discarded and a joint-to-joint mapping is used for the flexion/extension joints of the other three fingers and the thumb. On the other hand, a fingertip-position mapping is used to compute the mapped values of the abduction/adduction joints of the fingers and the thumb, and also the value of the thumb opposition joint.

\section{B. Motion analysis}

The synergies (i.e. couplings between DOFs) are obtained running a Principal Component Analysis (PCA) over the set of hand configurations mapped from the human movements. This returns a new basis of the hand configuration space, with the axes sorted in decreasing order of the associated sample variance (i.e. the first axis marks the direction with maximum sample variance and so on). Each axis is called a synergy and the motion along it, equivalent to a single DOF, implies the movement of several (or all) joints. Although nonlinear approaches to obtain synergies have been also proposed (e.g. [25]), the simple linear approximation of the PCA is enough to capture the subspace where the demonstrated motions lie, having been proved to be useful and implementable by a drive mechanism [26] and a real-time algorithm [14].

Two phases are observed in the mapped grasping motions (see Fig. 3). During the first phase, called pre-grasp phase, the trajectories of the hand joints are common motions opening the hand similarly in all the executions, regardless of the grasp type performed. Then, there is a certain moment in which the demonstrated trajectories begin to differ and specialize according to the type of grasp being carried out. This is the grasp phase itself. Nevertheless, the transition from one phase to the other is diffuse and does not occur at 


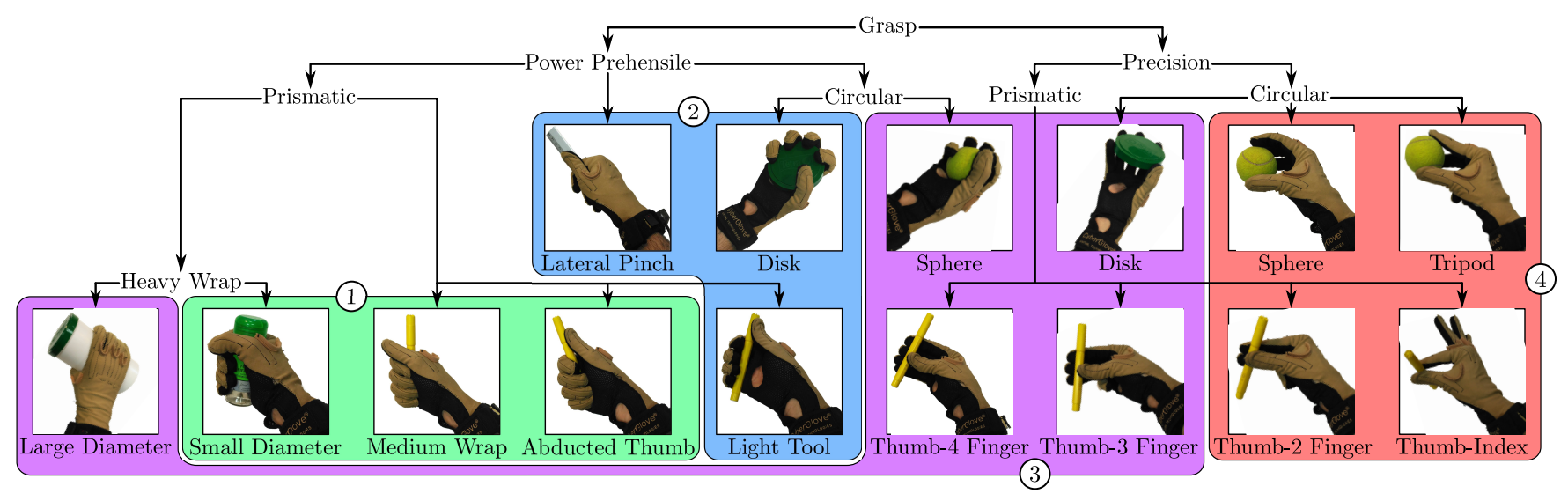

Fig. 2. The 15 force-closure grasps whose movements have been in this paper captured, classified, in a tree structure, adapting the grasp taxonomy of M.R. Cutkosky [22], and grouped into grasp families, 1 to 4, according to W. Dai, Y. Sun and X. Qian [24].

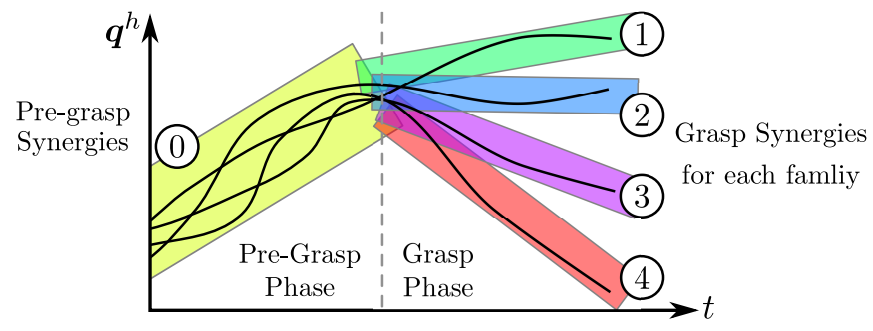

Fig. 3. Hypothetical mapped trajectories on the hand-configuration space, divided into pre-grasp and grasp phases, to obtain the common pre-grasp synergies (0) and the grasp synergies of each family (1 to 4).

the same time for all the demonstrations. Hence, the transition time is computed as follows. Let $Q$ be the set of hand configurations mapped from a given grasping demonstration, and, for a given time instant $t$, let $Q_{t}^{-}$and $Q_{t}^{+}$be the sets of configurations in $Q$ captured before and after $t$, respectively. In addition, let the likeness of the two given sets $Q_{A}$ and $Q_{B}$ of hand configurations be defined as the overlapping between the distributions of the configurations in the sets, which is a measure of the similarity between $Q_{A}$ and $Q_{B}$ [19]. This index can be computed as

$$
\mathcal{L}\left(Q_{A}, Q_{B}\right)=\frac{e^{-\frac{1}{2}\left(\boldsymbol{\mu}_{A}-\boldsymbol{\mu}_{B}\right)^{\top}\left(\Sigma_{A}+\Sigma_{B}\right)^{-1}\left(\boldsymbol{\mu}_{A}-\boldsymbol{\mu}_{B}\right)}}{\sqrt{(2 \pi)^{1+2 n}\left|\Sigma_{A}+\Sigma_{B}\right|}}
$$

where $\boldsymbol{\mu}_{A}$ and $\boldsymbol{\mu}_{B}$ are the barycenters and $\Sigma_{A}$ and $\Sigma_{B}$ are the covariance matrices of the configurations in $Q_{A}$ and $Q_{B}$, respectively. Then, the time instant $t$ indicating the transition between the two phases is defined as the one minimizing $\mathcal{L}\left(Q_{t}^{-}, Q_{t}^{+}\right)$. Thereby, the pre-grasp and grasp phases have been identified in the 180 mapped trajectories. On the one hand, all the pre-grasp phases have been grouped and used to compute the pre-grasp synergies. On the other hand, the grasp phases have been grouped according the grasp family which each demonstrated grasp belongs to, and, then, a set of grasp synergies has been computed for each grasp family (see Fig. 2). In this way, the pre-grasp synergies explain the hand motions in the pre-grasp phase in all the grasps, and each set of grasp synergies model the hand motions of each grasp family (Table I shows the accumulated sample variance for the obtained set of synergies).
TABLE I

ACCUMULATED SAMPLE VARIANCE AS A FUNCTION OF THE NUMBER $k$ OF CHOSEN SYNERGIES, FOR THE COMMON PRE-GRASP PHASE AND THE GRASP PHASE OF EACH OF THE DEMONSTRATED GRASP FAMILIES.

\begin{tabular}{|c||c|c|c|c|c|}
\hline \multirow{2}{*}{$k$} & \multirow{2}{*}{ Pre-Grasp } & \multicolumn{4}{|c|}{ Grasp Family } \\
\cline { 3 - 6 } & & 1 & 2 & 3 & 4 \\
\hline \hline 1 & $65.575 \%$ & $79.474 \%$ & $64.234 \%$ & $63.280 \%$ & $88.568 \%$ \\
\hline 2 & $77.795 \%$ & $86.125 \%$ & $81.877 \%$ & $84.238 \%$ & $91.955 \%$ \\
\hline 3 & $84.586 \%$ & $91.442 \%$ & $88.091 \%$ & $91.428 \%$ & $94.921 \%$ \\
\hline 4 & $90.316 \%$ & $94.015 \%$ & $92.225 \%$ & $94.377 \%$ & $\mathbf{9 6 . 6 0 6} \%$ \\
\hline 5 & $93.260 \%$ & $\mathbf{9 6 . 2 2 9} \%$ & $\mathbf{9 5 . 1 0 8} \%$ & $\mathbf{9 6 . 3 9 4} \%$ & $97.676 \%$ \\
\hline 6 & $\mathbf{9 5 . 9 9 6} \%$ & $97.665 \%$ & $96.781 \%$ & $97.664 \%$ & $98.685 \%$ \\
\hline 7 & $97.262 \%$ & $98.315 \%$ & $97.850 \%$ & $98.569 \%$ & $99.160 \%$ \\
\hline 8 & $98.165 \%$ & $98.802 \%$ & $98.834 \%$ & $99.027 \%$ & $99.449 \%$ \\
\hline 9 & $98.901 \%$ & $99.218 \%$ & $99.241 \%$ & $99.467 \%$ & $99.624 \%$ \\
\hline$\vdots$ & $\vdots$ & $\vdots$ & $\vdots$ & $\vdots$ & $\vdots$ \\
\hline 16 & $100 \%$ & $100 \%$ & $100 \%$ & $100 \%$ & $100 \%$ \\
\hline
\end{tabular}

For a robotic hand with $n$ DOFs, the synergies define an $n$-dimensional box centered at the barycenter of the configurations used to obtain the synergies and with each side aligned with a synergy [27]. So that the box contains the $(100-\alpha) \%$ of the configuration distribution (i.e. any hand configuration inside the box would be then similar to the ones used to compute the synergies), each side of this box measures $2 \sqrt{2} \operatorname{erf}^{-1}(\sqrt[n]{1-\alpha})$ times the standard deviation of the configurations in the corresponding direction. The dimension of the box can be reduced by using only $k<n$ synergies (picking them in order) such that $k$ is the minimum value making the accumulated variance be above a confidence level of $(100-\beta) \%$. In this work, $n$ is 16 and $\alpha=\beta=5 \%$ is considered. Thus, the dimension $k$ of the resulting lower-dimensional boxes, called $B_{k}$, is 4 or 5 for the grasp phase, depending on the grasp family (see bold values in Table I). For the pre-grasp phase, 6 synergies are needed (a little bit greater, as it was expected, since the movements of all the grasp families are explained in this case). Despite the simplification, the $B_{k}$ still represent accurately the mapped hand motions. Thereby, if the planning of the hand motions is performed in the corresponding $B_{k}$, the planning complexity is reduced and the obtained motions are similar to the movements mapped from the human operator. 


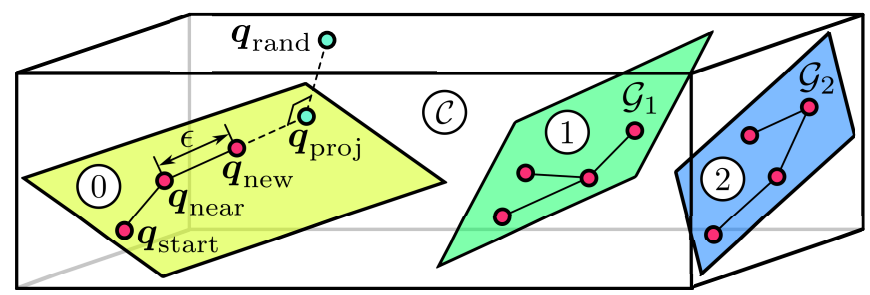

Fig. 4. Motion planning representation in $\mathcal{C}$-space: sample trees rooted at the start configuration $\boldsymbol{q}_{\text {start }}$ and the grasps $\mathcal{G}_{1}$ and $\mathcal{G}_{2}$, growing close the associated synergy lower-dimensional boxes ( 0 to 2 ), while steering a given configuration $\boldsymbol{q}_{\text {near }}$ towards a random $\boldsymbol{q}_{\text {rand }}$ and reaching $\boldsymbol{q}_{\text {new }}$. Note that the sample trees rooted at $\mathcal{G}_{1}$ and $\mathcal{G}_{2}$ belong to the same graph structure.

\section{Motion planning}

The proposed planner is based on the RRT-Connect [28], which is widely used in motion planning since it obtains good results even on robots with a high number of DOFs and with cluttered environments. However, it has been modified here to a) deal with multigoal queries, b) extend the sample trees following the synergies obtained above and, c) connect the sample trees in a less greedy fashion.

Let $\mathcal{C}$ be the robot configuration space, let $\boldsymbol{q} \in \mathcal{C}$ be a robot configuration, defined as an arm configuration $\boldsymbol{q}^{a}$ concatenated with a hand configuration $\boldsymbol{q}^{h}$, and let $\mathcal{G}=\left(\boldsymbol{q}^{h}, \boldsymbol{\chi}_{o}^{h}\right)$ be a grasp, composed of the hand configuration $\boldsymbol{q}^{h}$ and the object pose $\chi_{o}^{h}$ relative to the hand at the grasping time. Thereby, the introduced planner, outlined in Algorithm 1, is supplied with a collision-free start configuration $\boldsymbol{q}_{\text {start }}$ of the whole robot, the object pose $\chi_{o}^{r}$ relative to the robot, and a set $\left\{\mathcal{G}_{i}\right\}$ of grasps. The planner maintains two sample graphs, each one denoted by a pair formed by a set of edges $E$ and a set of vertices $V$. One of this graphs represents a sample tree rooted at the start configuration $\boldsymbol{q}_{\text {start }}$ (Line 1), and the other one contains the sample trees rooted each one at a grasp configuration (Lines 2-6), see Fig. 4. These grasp configurations are computed by, first, solving the arm inverse kinematics (IK) given the grasps $\mathcal{G}_{i}$ to perform and the object pose $\chi_{o}^{r}$ (Line 4) and, then, rejecting those cases which do not have an IK-solution or which imply collisions (Line 5). Thus, in each iteration, one of the graphs is steered towards a random configuration $\boldsymbol{q}_{\text {rand }}$ (uniformly sampled in $\mathcal{C}$ ), reaching a configuration $\boldsymbol{q}_{\text {new }}$ (Lines 7-8). Note that the STEER method, explained below, returns $\emptyset$ if the sample graph could not be extended, i.e. a collision is found. Next, the connection between the graphs is attempted. Notice that, in the classic RRT-Connect, the sample trees are connected greedily by extending one of trees directly until reaching the other tree or a collision. However, here in order to obtain an smoother connection, both graphs are, in alternation and successively, extended towards the last added configuration in the other graph (Lines 12-13), until the graphs are connected and, then, the found solution path is returned (Line 10). In case the steering process fails (Line 9), the sample graphs swap their roles (Line 14) and the whole process is repeated until a solution is found or some termination condition is satisfied (Line 6), e.g. surpassing a maximum planning time, number of iterations or memory allocation.
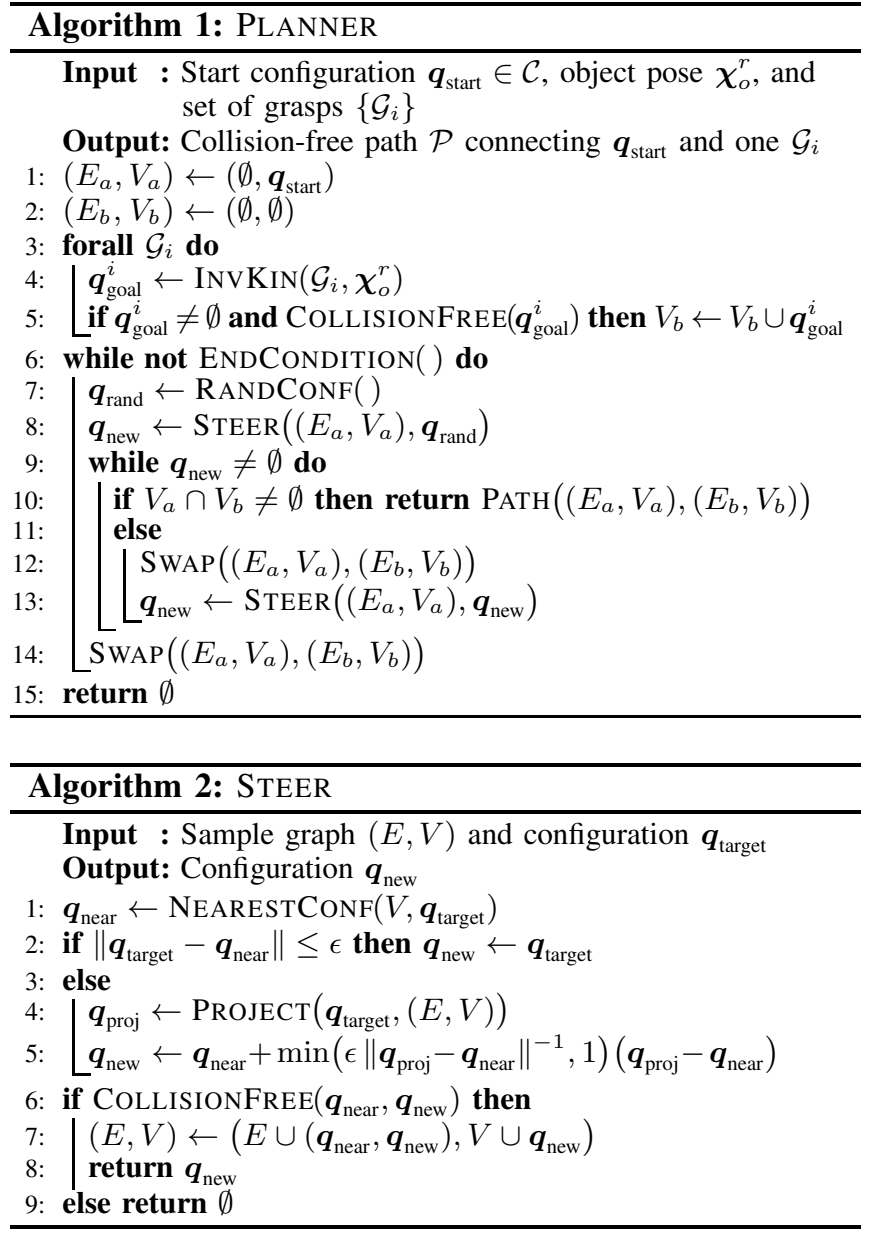

In order to integrate the synergies into the motion planning, the standard function extending the sample tree in RRT-based planners is replaced here by the function STEER, described in Algorithm 2. As in the classic method, a single step is performed from $\boldsymbol{q}_{\text {near }}$, the configuration in the graph closest to the desired target configuration $\boldsymbol{q}_{\text {target }}$ (Line 1), reaching a new configuration $\boldsymbol{q}_{\text {new }}$. If the segment connecting $\boldsymbol{q}_{\text {near }}$ and $\boldsymbol{q}_{\text {new }}$ is collision-free, the segment is added to the graph and $\boldsymbol{q}_{\text {new }}$ is returned (Lines 6-8). Otherwise, $\emptyset$ is returned (Line 9). However, here, $\boldsymbol{q}_{\text {new }}$ is computed differently, i.e. following the synergies instead. Thereby, if $\boldsymbol{q}_{\text {target }}$ is close to $\boldsymbol{q}_{\text {near }}, \boldsymbol{q}_{\text {new }}$ is $\boldsymbol{q}_{\text {target }}$ (Line 2), so that in the event that the two sample graphs are close to be connected, the guideline to follow the synergies may be relaxed. Otherwise, a step, with a maximum length $\epsilon$, is taken not towards the desired $\boldsymbol{q}_{\text {target }}$ (as it would be done in the standard procedure) but towards its projection $\boldsymbol{q}_{\text {proj }}$ onto the lower-dimensional box spanned by the synergies (Lines 4-5), see Fig. 4. Note that, in the PROJECT procedure, the arm component of $\boldsymbol{q}_{\text {target }}$ remains the same and the hand component is projected onto the lower-dimensional box $B_{k}$ of synergies associated with the root of the sample tree containing $\boldsymbol{q}_{\text {near }}$ (i.e. if $\boldsymbol{q}_{\text {near }}$ belongs to the sample tree rooted at $\boldsymbol{q}_{\text {start }}, \boldsymbol{q}_{\text {target }}$ is projected onto the box of pre-grasp synergies; otherwise, $\boldsymbol{q}_{\text {near }}$ belongs to a sample tree rooted at a certain $\mathcal{G}_{i}$ and, hence, $\boldsymbol{q}_{\text {target }}$ is projected onto the box of synergies associated with $\mathcal{G}_{i}$ ). 

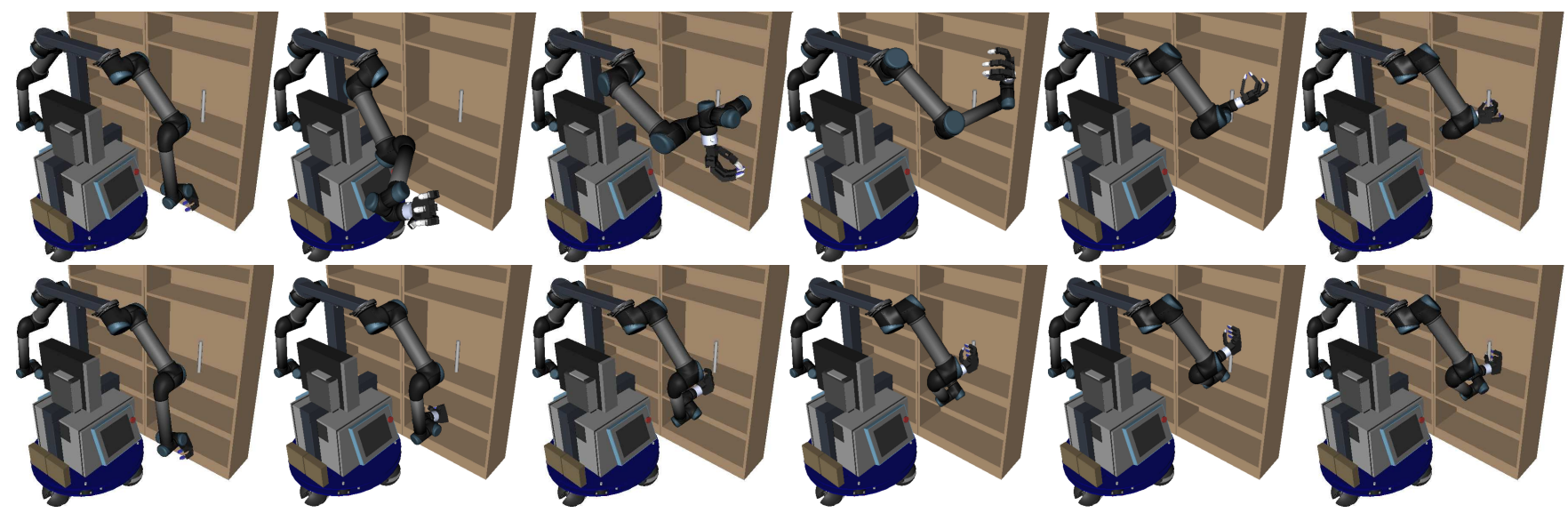

Fig. 5. Snapshots of paths obtained with an standard RRT-Connect (top), and the proposed procedure using the proper synergies for each grasp type (bottom).


Fig. 6. Examples of different grasp types used within the motion planning, each one of a different grasp family: Thumb-2 Finger, Thumb-3 Finger, Medium Wrap and Lateral Pinch (from left to right, respectively).

\section{VALidATION OF THE APPROACH}

For illustrative purposes, the motions of an anthropomorphic dual-arm robot have been planned (see Fig. 1), with the robot located in front of a bookshelf and, starting off from a natural standing pose, must grasp a cylinder standing on one of the shelves (see Fig. 5). Besides, the robot must perform human-like motions while avoiding the collisions with itself, the bookshelf and the cylinder. For this, the planning algorithm is provided with the exact position of the cylinder and with a set of different force-closure grasps $\mathcal{G}_{i}$ (from different grasp types and grasp families, see Fig. 6). This information can be obtained, for instance, from the vision system on the robot and a grasp generator, respectively. In order to evaluate and compare the performance of the proposed approach, three planners have been benchmarked:

a) A standard RRT-Connect, modified to tackle multi-goal queries, planning without using synergies.

b) The proposed approach, planning using the proper grasp synergies in relation of the grasp type to be performed.

c) The proposed approach, but in this case the grasp synergies and the grasp families have been intentionally mismatched (i.e. each grasp family has been randomly associated with the synergies of another grasp family).

Note that, at every planner execution, the orientation and position of the robot are lightly modified at random and that a different set of 8 grasps $\mathcal{G}_{i}$ are randomly selected from the set of precomputed grasps. Thereby, the planners are provided with a single start configuration and a set of 8 different goal configurations, i.e. one per each of the selected grasps.
TABLE II

AVERAGE RESUlTS OF THE MOTION PLANNING WHEN RUNNING THE CLASSIC RRT-CONNECT (a) AND THE PROPOSED APPROACH WITH THE PROPER (b) AND WITH MISMATCHED GRASP SYNERGIES (c).

\begin{tabular}{|c||c|c|c|c|c|c|c|}
\hline \multirow{2}{*}{} & $\begin{array}{c}\text { Success } \\
\text { rate }\end{array}$ & $\begin{array}{c}\text { Planning } \\
\text { time }\end{array}$ & $\begin{array}{c}\text { \# ite- } \\
\text { rations }\end{array}$ & $\begin{array}{c}\text { \# collision } \\
\text { checks }\end{array}$ & $\begin{array}{c}\text { Valid } \\
\text { segments }\end{array}$ & $\begin{array}{c}\text { Path } \\
\text { length }\end{array}$ & $\begin{array}{c}\text { Human- } \\
\text { likeness }\end{array}$ \\
\hline $\mathrm{a}$ & $97 \%$ & $51.80 \mathrm{~s}$ & 1834 & 32231 & $68.3 \%$ & $14.18 \mathrm{rad}$ & $73.6 \%$ \\
\hline $\mathrm{b}$ & $100 \%$ & $6.21 \mathrm{~s}$ & 274 & 10649 & $80.0 \%$ & $7.79 \mathrm{rad}$ & $83.1 \%$ \\
\hline $\mathrm{c}$ & $100 \%$ & $11.79 \mathrm{~s}$ & 484 & 13667 & $75.3 \%$ & $8.35 \mathrm{rad}$ & $81.9 \%$ \\
\hline
\end{tabular}

The experiments introduced above have been implemented within the environment The Kautham Project [29], a motion planning and simulation environment developed at the Institute of Industrial and Control Engineering (IOC-UPC) for teaching and research purposes, and run in a $2.13-\mathrm{GHz}$ Intel 2, 4-GB RAM PC. A maximum planning time of $100 \mathrm{~s}$ is considered for each planner instance. Thereby, if a path is not found within this time, the execution is marked as a failure. After 100 executions, Table II shows the average values of the success rate, the planning time, the number of iterations and collision checks, the rate of valid segments (i.e. the ratio of iterations in which the sample trees actually grow), the path length (measured in $\mathcal{C}$ as the weighted sum of accumulated joint movements along the path), and the path human-likeness. The human-likeness index computes the misalignment of a path with respect to some given reference human movements [27]. Here, natural free-movements of the operator while moving freely the fingers in an unconstrained way (i.e. without performing any specific task), trying to cover the whole hand workspace, are used as a reference.

On the one hand, it can be noticed from the simulation results that effectively the proposed planning approach is several times faster than the standard RRT-Connect algorithm (up to an order of magnitude). In fact, the motion planning can be solved within the time restrictions for the $100 \%$ of the executions only when the proposed approach is used, either when the grasp synergies are properly associated with the selected grasps or when they are mismatched. It can be stated that the use of synergies clearly reduces the planning time since the solution is enforced to lie close to the lower-dimensional boxes $B_{k}$. This focuses the search 
efforts close to the demonstrated movements (which belong to a set of demonstrated feasible solutions), thus accelerating the connection of the sample trees and, thereby, reducing the needed number of iterations and collision checks to find a solution. In addition, since the grasp synergies are obtained from feasible movements, the probability of obtaining collision-free robot configurations increases when using synergies (see valid segments rate in Table II), reducing greatly the computation time. The results also show that even when not the correct grasping synergies are used, i.e. case $c$, the benefits of using synergies are still evident. In this case, the planning time is slightly penalized, however, it is still a better option than not using synergies at all.

On the other hand, the proposed planning procedure produces movements of the robotic system that look more natural and human-like (see human-likeness in Table II), since the grasp synergies are obtained from human demonstrations and the human-likeness is preserved within the planning process. Besides of the numerical results, the higher human-likeness of the proposed approach can be noticed in Fig. 5, which shows representative solution paths for cases $a$ and $b$.

\section{CONClusions And Future Work}

This paper has proposed a procedure to efficiently obtain human-like hand-arm movements to grasp a given object. To this end, the movements of a human operator performing different grasps on different objects have been captured and mapped to the robot. These grasp movements have been classified according to a grasp taxonomy, and for each grasp family a set of human-demonstrated synergies (couplings between DOFs) have been computed. In addition, a pre-grasp set of synergies has also been computed, common for all the grasp families. Finally, a motion planner profiting from these synergies has been presented and compared against other state-of-the-art planners planning the motions of a real anthropomorphic dual-arm robot. The effect of using the grasp synergies, even when they are not the ones associated with the grasp being performed, has been also investigated, producing good results in both cases.

In the future we plan to supplement the current results by incorporating into the study the movements of several human operator, to understand if the behavior is any different depending on age, gender, etc. Besides, the proposal opens several interesting potential research lines, such as its extension to the velocity space and the coordination of the robot base, arms and hands all at the same time.

\section{REFERENCES}

[1] T. Fukuda, R. Michelini, V. Potkonjak, S. Tzafestas, K. Valavanis, and M. Vukobratovic. How far away is "artificial man". IEEE Robotics and Automation Mag., 8(1):66-73, Mar. 2001.

[2] M. Elbanhawi and M. Simic. Sampling-based robot motion planning: A review. IEEE Access, 2:56-77, Jan. 2014.

[3] L.E. Kavraki, P. Svestka, J.-C. Latombe, and M.H. Overmars. Probabilistic roadmaps for path planning in high-dimensional configuration spaces. IEEE Trans. Robotics and Autom., 12(4):566-580, Aug. 1996.

[4] J.J. Kuffner and S. LaValle. RRT-Connect: An efficient approach to single-query path planning. In Proc. IEEE Int. Conf. Robotics and Automation, pages 995-1001, Apr. 2000.
[5] M. Stilman. Global manipulation planning in robot joint space with task constraints. IEEE Trans. Robotics, 26(3):576-584, June 2010.

[6] N. García, R. Suárez, and J. Rosell. HG-RRT*: Human-Guided optimal random trees for motion planning. In Proc. IEEE Int. Conf. Emerging Technologies and Factory Automation, Sept. 2015.

[7] J. Lee, O. Kwon, L. Zhang, and S.E. Yoon. A selective retractionbased rrt planner for various environments. IEEE Trans. Robotics, 30(4):1002-1011, Aug. 2014.

[8] B.D. Argall, S. Chernova, M. Veloso, and B. Browning. A survey of robot learning from demonstration. Robotics and Auton. Systems, 57(5):469-483, May 2009.

[9] L. Colasanto, R. Suaŕez, and J. Rosell. Hybrid mapping for the assistance of teleoperated grasping tasks. IEEE Trans. Systems, Man and Cybernetics, 43(2):390-401, 2013.

[10] R. Suárez, J. Rosell, and N. García. Using synergies in dual-arm manipulation tasks. In Proc. IEEE Int. Conf. Robotics and Automation, pages 5655-5661, May 2015.

[11] M. Santello, M. Flanders, and J.F. Soechting. Patterns of hand motion during grasping and the influence of sensory guidance. $J$. Neuroscience, 22(4):1426-1435, Feb. 2002.

[12] M.T. Ciocarlie and P.K. Allen. Hand posture subspaces for dexterous robotic grasping. Int. J. Robotics Research, 28(7):851-867, July 2009.

[13] F. Ficuciello, G. Palli, C. Melchiorri, and B. Siciliano. Postural synergies of the UB Hand IV for human-like grasping. Robotics and Auton. Systems, 62(4):515-527, Apr. 2014.

[14] T. Wimböck, B. Jan, and G. Hirzinger. Synergy-level impedance control for a multifingered hand. In Proc. IEEE/RSJ Int. Conf. Intelligent Robots and Systems, pages 973-979, Sept. 2011.

[15] M. Bianchi, P. Salaris, and A. Bicchi. Synergy-based hand pose sensing: Reconstruction enhancement. Int. J. Robotics Research, 32(4):396-406, Apr. 2013.

[16] S. Sun, C. Rosales, and R. Suárez. Study of coordinated motions of the human hand for robotic applications. In Proc. IEEE Int. Conf. Information and Automation, pages 776-781, June 2010.

[17] M. Gabiccini, A. Bicchi, D. Prattichizzo, and M. Malvezzi. On the role of hand synergies in the optimal choice of grasping forces. Autonomous Robots, 31:235-252, July 2011.

[18] D. Prattichizzo, M. Malvezzi, and A. Bicchi M. Gabiccini. On motion and force controllability of precision grasps with hands actuated by soft synergies. IEEE Trans. Robotics, 29(6):1440-1456, Dec. 2013.

[19] N. García, R. Suárez, and J. Rosell. Task-Dependent synergies for motion planning of an anthropomorphic dual-arm system. IEEE Trans. Robotics, 33(3):756-764, June 2017.

[20] N. García, J. Rosell, and R. Suárez. Motion planning using firstorder synergies. In Proc. IEEE/RSJ Int. Conf. Intelligent Robots and Systems, pages 2058-2063, Sept. 2015.

[21] N. García, R. Suárez, and J. Rosell. First-Order synergies for motion planning of anthropomorphic dual-arm robots. In Proc. IFAC World Congress, pages 2283-2290, July 2017.

[22] M.R. Cutkosky. On grasp choice, grasp models, and the design of hands for manufacturing tasks. IEEE Trans. Robotics and Automation, 5(3):269-279, June 1989.

[23] T. Feix, J. Romero, H. B. Schmiedmayer, A. M. Dollar, and D. Kragic. The GRASP taxonomy of human grasp types. IEEE Trans. HumanMachine Systems, 46(1):66-77, Feb. 2016.

[24] W. Dai, Y. Sun, and X. Qian. Functional analysis of grasping motion. In IEEE/RSJ Int. Conf. Intelligent Robots and Systems, pages 35073513, Nov. 2013.

[25] J. Romero, T. Feix, C.H. Ek, H. Kjellström, and D. Kragic. Extracting postural synergies for robotic grasping. IEEE Trans. Robotics, 29(6):1342-1352, Dec. 2013

[26] W. Chen, C. Xiong, and S. Yue. Mechanical Implementation of Kinematic Synergy for Continual Grasping Generation of Anthropomorphic Hand. IEEE/ASME Trans. Mechatronics, 20(3):1249-1263, June 2015.

[27] N. García, J. Rosell, and R. Suárez. Motion planning by demonstration with human-likeness evaluation for dual-arm robots. IEEE Trans. Systems, Man, and Cybernetics: Systems, PP(99):1-10, 2017.

[28] J. J. Kuffner and S. LaValle. RRT-Connect: An efficient approach to single-query path planning. In Proc. IEEE Int. Conf. Robotics and Automation, pages 995-1001, Apr. 2000.

[29] J. Rosell, A. Pérez, A. Aliakbar, Muhayyuddin, L. Palomo, and N. García. The Kautham project: A teaching and research tool for robot motion planning. In Proc. IEEE Int. Conf. Emerging Technologies and Factory Automation, Sept. 2014. 